\title{
Transmission of PsV-F and PsV-S Mycoviruses during Conidiogenesis of Penicillium stoloniferum
}

\author{
By D. M. DeMARINI, C. P. KURTZMAN, D. I. FENNELL, K. A. WORDEN \\ AND R. W. DETROY \\ Northern Regional Research Center, Agricultural Research Service, \\ U.S. Department of Agriculture, Peoria, Illinois 61604, U.S.A.
}

(Received 17 November 1976)

\begin{abstract}
SUM MAR Y
Levels of both fast and slow Penicillium stoloniferum virus (PsV-F and PsV-S) were evaluated in single conidial isolates of $P$. stoloniferum NRRL5267. Approximately $7 \%(3 / 43)$ of a random population of conidia from a culture of $P$. stoloniferum contained no PsV-F, while other conidia produced cultures with various levels of PsV-F. The greatest variation in PsV-F levels was observed in single conidial isolates from cultures that contained low to intermediate levels of virus, i.e. I I to $25 E_{26 i \prime}^{1 \mathrm{~cm}}$ units per g dry weight of mycelium. The least variation was seen in isolates from a culture with a high PsV-F level ( $37 E_{260}$ units per g dry weight of mycelium). Two of the original 43 single conidial isolates contained no detectable PsV-F or PsV-S. Of the second-generation single conidial isolates from an original PsV-S ${ }^{+}$ PsV-F- isolate, $6 \%$ failed to show detectable PsV-S.

PsV-F levels of cultures remained constant throughout a series of transfers when the inoculum was a mixture of conidia and mycelium. The presence of PsV-F at different levels, or its complete absence, did not affect fungal biomass, conidium size, morphology or viability. The results are discussed in terms of how conidiogenesis may influence the transmission or persistence of PsV-F and PsV-S during morphogenesis.
\end{abstract}

\section{INTRODUCTION}

The presence of polyhedral virus-like particles in Penicillium stoloniferum Thom was first noted by Ellis \& Kleinschmidt (1967). Further investigations revealed two electrophoretically and serologically distinct double-stranded RNA viruses associated with $P$. stoloniferum NRRL5267 (Banks et al., 1968; Buck \& Kempson-Jones, 1973). The virus with the higher electrophoretic mobility (PsV-F) contains three distinct molecular weight classes of doublestranded RNA whereas the slower virus (PsV-S) contains two double-stranded RNA classes (Bozarth, Wood \& Mandelbrot, 1971).

PsV-F and PsV-S have been isolated and characterized from mycelia (Detroy, Lillehoj \& Hesseltine, 1974) and from conidia of $P$. stoloniferum (Sansing et al., 1973). Using in vivo fluorescent-antibody techniques, Adler \& MacKenzie (1972) observed that both viruses occur in the cytoplasm of the hyphae and are not compartmentalized. Viruses have also been seen throughout the cytoplasm in thin sections of conidia of $P$. stoloniferum (Hooper et al., 1972). Recently, PsV-F- isolates of $P$. stoloniferum were obtained from conidia irradiated with ultraviolet (u.v.) light (Still, Detroy \& Hesseltine, 1975).

To understand the distribution of virus within cultures of $P$. stoloniferum and how certain distributions might influence virus levels in single conidial (SC) isolates, we analysed the PsV-F and PsV-S levels in 43 SC isolates and in second-generation SC isolates derived from four of these original cultures. 


\section{METHODS}

Organism and growth conditions. The virus-containing strain of Penicillium stoloniferum Thom NRRL5267 (ATCCI 4586) was from the ARS Culture Collection, Northern Regional Research Center. Using a micromanipulator, 43 random conidia were selected from a suspension prepared from a 7-day-old M40Y slant [containing ( $\%$, w/v): sucrose, 40; malt, 2; yeast extract, 0.5 ; and agar, 2]. Single conidia were placed on slants of potato/ sucrose agar (containing infusion from $200 \mathrm{~g}$ potatoes $1^{-1}, 2 \%$ sucrose and $2 \%$ agar) and incubated at $24{ }^{\circ} \mathrm{C}$. All subsequent mass transfers and SC isolates were grown on slants of $\mathrm{M} 40 \mathrm{Y}$ and incubated at $28{ }^{\circ} \mathrm{C}$. Cultures for virus analyses were grown by inoculating $75 \mathrm{ml}$ YED medium (containing $2 \%$ yeast extract and $5 \%$ dextrose) in a $300 \mathrm{ml}$ Erlenmeyer flask with $\mathrm{I} \mathrm{ml}$ of a suspension of $10^{7}$ conidia in $0.01 \%(\mathrm{v} / \mathrm{v})$ Triton $\mathrm{X}$. The cultures were incubated at $28{ }^{\circ} \mathrm{C}$ on a Brunswick shaker at 250 rev. $\min ^{-1}$ for $72 \mathrm{~h}$.

Preparation and measurement of PSV-F and PsV-S. Mycelia were harvested by vacuum filtration and $\mathrm{I} g$ (wet weight) samples were transferred to $75 \mathrm{ml}$ glass Bronwill mechanical cell homogenizer flasks containing $50 \mathrm{ml} 0.1 \mathrm{M}$-phosphate buffer $\mathrm{pH} 7.2$ and $45 \mathrm{~g}$ of $\mathrm{I} \mathrm{mm}$ glass beads. The samples were homogenized for $3 \mathrm{~min}$ at $4000 \mathrm{rev} . \mathrm{min}^{-1}$ under a stream of $\mathrm{CO}_{2}$ at $0{ }^{\circ} \mathrm{C}$. The homogenates were centrifuged at $8000 \mathrm{~g}$ for $\mathrm{I}$ o min to remove cell debris, and the resulting supernatant fluid was centrifuged at $105000 \mathrm{~g}$ for $2 \mathrm{~h}$. The pellet was resuspended in $\mathrm{I} \mathrm{ml}$ phosphate buffer, centrifuged at $8000 \mathrm{~g}$ for Io $\mathrm{min}$, and the resulting supernatant fluid was then analysed for virus. All analyses were based on duplicate cultures.

Samples of the virus preparation were layered on to polyacrylamide gels [containing $2.4 \%(\mathrm{w} / \mathrm{v})$ acrylamide, $0.15 \%(\mathrm{w} / \mathrm{v})$ bisacrylamide and $0.5 \%(\mathrm{w} / \mathrm{v})$ agarose $)$ in TAE-SDS buffer, $\mathrm{pH} 7.0$ [containing $0.09 \mathrm{M}$-Tris, $0.07 \mathrm{M}$-sodium acetate, $0.002 \mathrm{M}$-EDTA and $0.5 \%$ $(\mathrm{w} / \mathrm{w})$ sodium dodecyl sulphate] prepared essentially as described by Loening (I967). Electrophoresis was performed for $2 \mathrm{~h}$ at $7 \mathrm{~mA}$ per tube at $25^{\circ} \mathrm{C}$. A Gilford linear transport system was used to scan the gels at $260 \mathrm{~nm}$. A PsV-F standard curve was prepared because the area under the PsV-F curve shows a linear relationship to the amount of PsV-F applied between 0.03 and $0.25 E_{260}^{\mathrm{L} \mathrm{cm}}$ units of standard PsV-F.

The presence or absence of PsV-S was determined by comparing the gel electrophoretograms with that of a standard preparation containing both viruses. Levels of PsV-S were estimated in $E_{260}$ units compared with known quantities of PsV-F.

\section{RESULTS}

As indicated in Fig. I, PsV-F was the preponderant virus in P. stoloniferum NRRL5267. PsV-S occurred at low titres and showed instability. Analyses of vegetative tissue generally indicated a I0- to 20-fold difference between PsV-F and PsV-S levels, as in Fig. I.

Preliminary analyses of the original $43 \mathrm{SC}$ isolates disclosed six that were of particular interest because of their differing titres of PsV-F and PsV-S (Fig. 2). The other $37 \mathrm{SC}$ isolates contained 20 to $29 E_{260}$ units of PsV-F virus per flask, and various levels of PsV-S. In isolates SC-7 and SC-IO, neither PsV-F nor PsV-S was detected by gel electrophoresis. Isolate SC-I 9 contained no PsV-F, but 0.1 to $0.4 E_{260}$ units of PsV-S per flask were detected. Isolate SC-30 contained $25 E_{260}$ units of PsV-F per flask and very low levels of PsV-S, while SC-37 and SC-40 contained 45 and I $_{5} E_{260}$ units of PsV-F per flask, respectively, and $0 \cdot I$ to $0.5 E_{260}$ units of PsV-S per flask.

Isolates $\mathrm{SC}-19,30,37$ and 40 were selected as representatives for further conidial selection because of their different titres of PsV-F (o to $50 E_{260}$ units per flask) and PsV-S (o to $0.5 E_{260}$ 


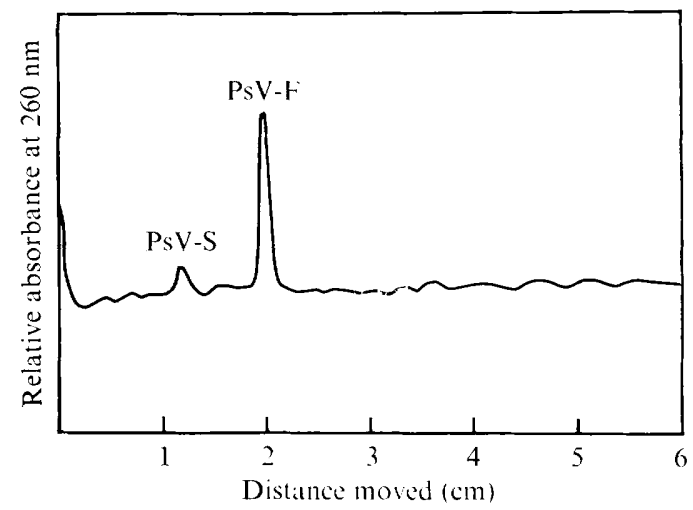

Fig. I. Electrophoretogram of PsV-S and PsV-F from $P$. stoloniferum tissue.

units per flask). A number of conidia ( 35 to 43 ) were selected randomly from each representative isolate. Figure 2 indicates the genealogy of the conidia and their PsV-F and PsV-S status.

All of the $35 \mathrm{SC}$ isolates from SC-I 9 that were investigated were devoid of PsV-F. However, all but two isolates (SC-19-3I and SC-19-34) contained PsV-S. Analyses of 36 SC isolates from SC-30, which had an intermediate PsV-F titre, revealed 34 with intermediate levels of PsV-F and variable contents of PsV-S, one (SC-30-II) with a high level of PsV-F and detectable PsV-S, and one (SC-30-12) with no PsV-F but detectable PsV-S. The 34 SC isolates from SC-37 had high to intermediate PsV-F levels and variable levels of PsV-S $\mathrm{SC}$ isolates from SC-40 had a wide range of PsV-F titres as shown by SC-40-30 (high), SC-40-49 (low) and SC-40-43 (none).

Although virus levels varied from isolate to isolate, the biomass of all cultures was approximately the same, as was the viability of all isolates except culture 40 which exhibited

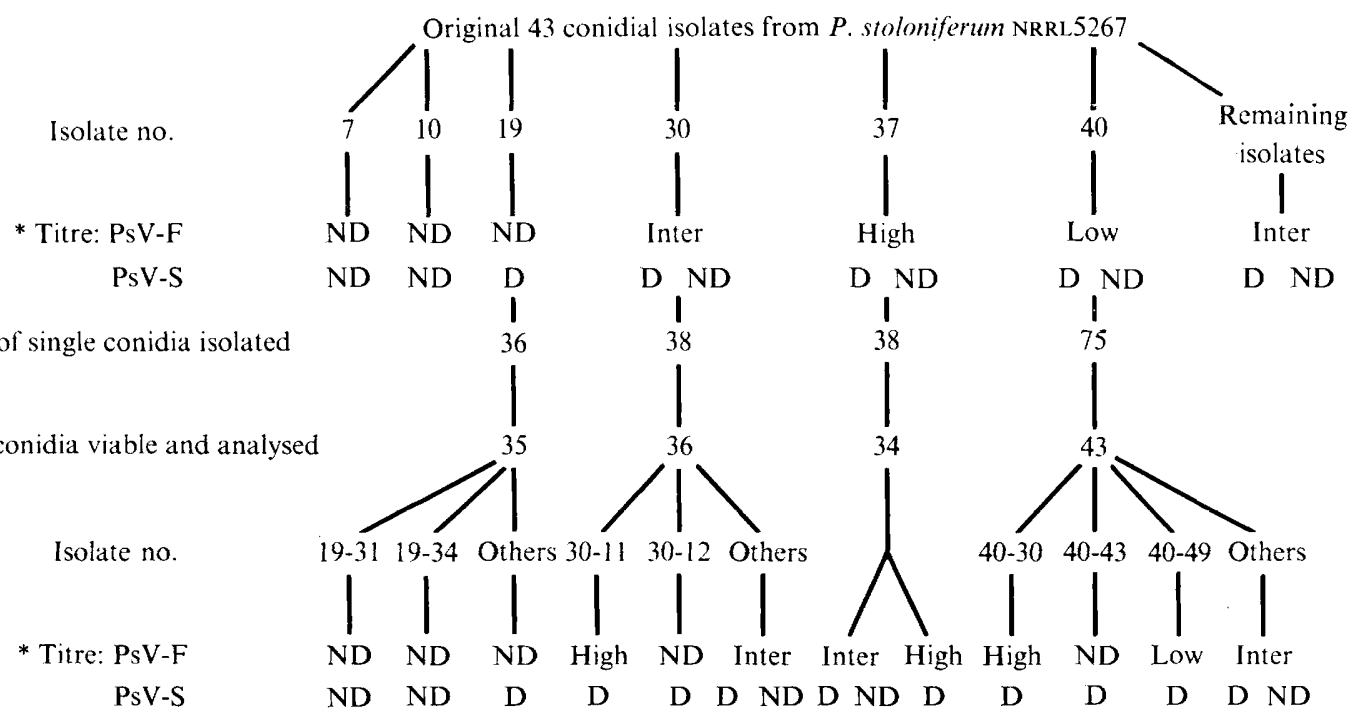

Fig. 2. Genealogy of conidial isolates of $P$. stoloniferum.

* Range of titre (in $E_{260}$ units per flask): ND, none detected; D, detectable, 0.I to 0.9;

Low, I to I9; Inter(mediate), 20 to 29 ; High, 30 to 90. 
Table I. Growth characteristics of single conidial isolates

$\begin{array}{rccc}\text { Isolate } & \begin{array}{c}\text { Conidial } \\ \text { viability }(\%)\end{array} & \text { Original } & \begin{array}{c}\text { Biomass of SC isolate } \\ \text { (g dry wt per flask) }\end{array} \\ \text { SC-19 } & 97 & 0.953 & \text { 2nd generation } \\ \text { SC-30 } & 92 & 0.962 & 0.806 \\ \text { SC-37 } & 92 & 0.962 & 0.958 \\ \text { SC-40 } & 57 & 0.973 & \mathrm{I} \cdot 003\end{array}$

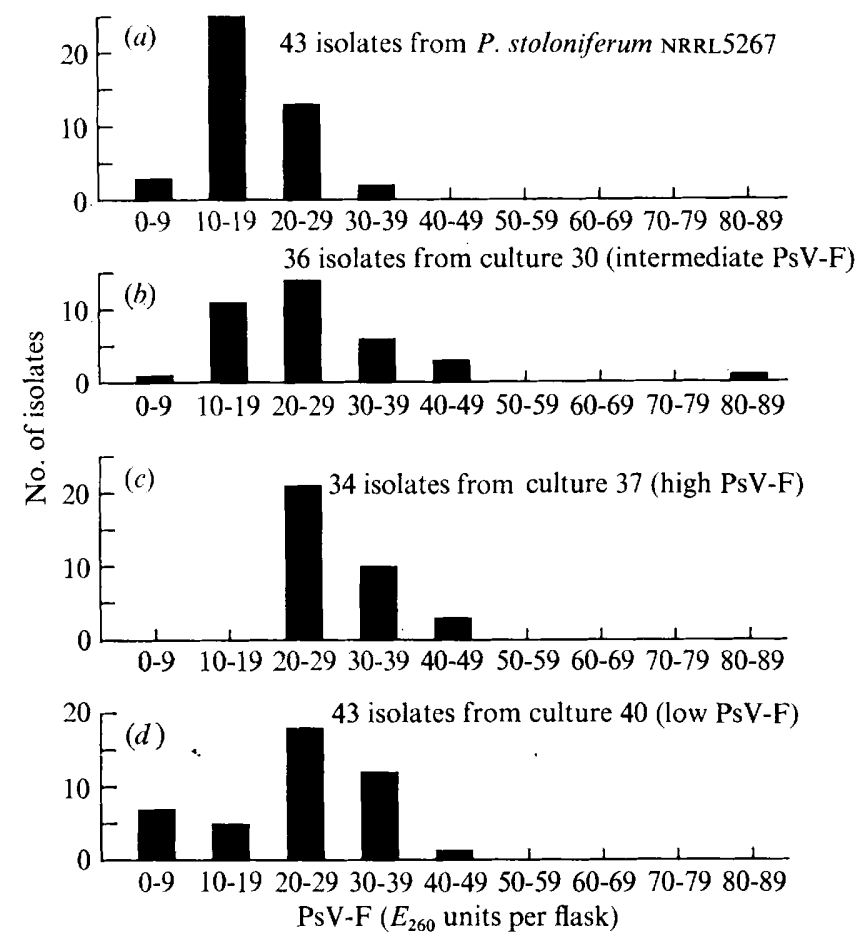

Fig. 3. Histograms of PsV-F levels in: (a) SC isolates from the original $P$. stoloniferum NRRL5267 culture; $(b, c, d) \mathrm{SC}$ isolates from three $\mathrm{SC}$ isolates derived from the original $P$. stoloniferum culture.

low viability (Table I). No cultural or morphological differences were observed in the original $43 \mathrm{SC}$ isolates.

Figure 3 shows the distribution of PsV-F levels both in SC isolates of the original $P$. stoloniferum culture and in second-generation $\mathrm{SC}$ isolates from three of the original ones. The PsV-F levels ( $E_{260}$ units per flask) of SC-37, 30 and 40 were high (35), intermediate (26) and low (IO), respectively. The histograms demonstrate that the PsV-F profile of a series of $\mathrm{SC}$ isolates from a particular culture reflects the PsV-F level of that culture. All cultures showed considerable variation in PsV-F levels of SC isolates; the greatest variation was in $\mathrm{SC}$ isolates from cultures that contained low to intermediate levels of virus, i.e. SC-40 and 30, and the least, in isolates from culture 37 which had a high level of PsV-F. PsV-S, when detected, was low in all $\mathrm{SC}$ isolates examined.

Although cultures derived from single conidia varied from one to another in PsV-F level, 
mass transfer of conidia permitted maintenance of stock cultures of SC isolates in which PsV-F levels remained constant.

\section{DISCUSSION}

The similarity of the biomass of the isolates shows that the presence of PsV-F and/or $\mathrm{PsV}-\mathrm{S}$ at different levels or its absence does not alter the growth of the fungus. Also, the presence of PsV-F or PsV-S produced no detectable morphological changes in the conidia or the vegetative culture. Furthermore, conidia from isolates with either high levels of PsV-F or PsV-S or no viruses were equally viable.

Mass transfer of a large population of conidia $\left(10^{6}\right.$ to $\left.10^{7}\right)$ or mycelial fragments from a particular isolate eliminates variability and results in a culture with virus levels similar to that of the original SC isolate.

Virus-like particles have not been observed in the rapidly extending tips of actively growing hyphae of virus-infected cultures (Hooper et al., 1972; Border et al., 1972), whereas older hyphae contain many virus particles. This characteristic was used in obtaining virusfree subcultures of a virus-containing strain of Agaricus bisporus by hyphal-tip isolations (Hollings \& Stone, I97I). If a similar condition prevails in the developing penicillus, the variation in both PsV-F and PsV-S levels shown by the different SC isolates may be explained by the process of conidiogenesis in Penicillium (Fletcher, I97 I Cole \& Kendrick, I969; Zachariah \& Fitz-James, I967).

Phialides, the sporogenous cells from which the catenate conidia are produced in basipetal succession, arise asynchronously at the apex of their subtending conidiophore or metula. On a suitable medium, conidium production begins as soon as the first phialide is formed. The protoplast of a conidium initial in the expanding tip of a phialide is continuous with that of the phialide. Shortly before the developing conidium emerges, the two protoplasts are separated by a thin perforate septum that permits continued transfer of cytoplasm and nutrients. Within minutes after the conidium bursts through the tip of the phialide, its cytoplasmic connection with the phialide is severed by plugging of the pore in the thickening septum. Should this separation occur before the infecting viruses advance into the developing conidium, a virus-free conidium may result. Subsequent conidia from the same phialide, or from other phialides in the same penicillus, might well contain different concentrations of functional or non-functional viruses.

Obviously, this theory may be tested by isolation of conidia in sequence from a single phialide. Various techniques have been applied in efforts to obtain a preparation suitable for this operation; to date, none has succeeded.

Our results demonstrate that $7 \%$ of the original SC isolates did not contain PsV-F and $5 \%$ harboured no virus particles whatsoever. Subsequent analysis of a large number of SC isolates derived from original SC isolates with high and intermediate PsV-F levels, showed that 5 to $10 \%$ contained only PsV-S or no viruses. Analyses of SC isolates derived from a strain containing only PsV-S revealed that $6 \%$ failed to show detectable PsV-S.

This loss of ability to support PsV-F or PsV-S replication suggests that the conidiation process may be involved. Lack of transmission of mature replicating virus particles to developing conidia might explain the variation in virus level or complete absence of detectable virus in $\mathrm{SC}$ isolates.

The PsV-S and PsV-F strains obtained by SC isolation should be genetically compatible with the PsV-F-containing strain from which they were derived and, therefore, useful in forming heterokaryons for mycovirus infection experiments. Lhoas (1971) obtained limited infection through heterokaryosis, a process that depends on compatibility between donor and recipient strains. 
Volterra et al. (1975) reported that the distribution of two viruses of Penicillium citrinum differed in sporogenic and asporogenic segregants of the culture. We have also noted asporogenic segregants in isolates of $P$. stoloniferum. However, our preliminary investigation does not suggest that the presence of PsV-F or PsV-S at different levels, or their absence, accounts for these segregants. The demonstration of PsV-F- ${ }^{-}$and PsV-S- conidia in nonirradiated cultures of $P$. stoloniferum may rule out replication of these viruses by the host genome as suggested by Still et al. (1975) who isolated u.v. mutants lacking PsV-F, but harbouring PsV-S.

\section{REFERENCES}

AdLeR, J. P. \& MACKenzIE, D. W. (1972). Fungal viruses: intrahyphal localization of Penicillium stoloniferum viruses by fluorescent-antibody. In Abstracts of the Annual Meeting of the American Society for Microbiology, p. 68. Washington: American Society for Microbiology.

Banks, G. T., Buck, K. W., Chain, E. B., Himmelweit, F., Marks, J. E., Tyler, J. M., Hollings, A., Last, F. T. \& STONE, O. M. (I968). Viruses in fungi and interferon stimulation. Nature, London 218, 542-545.

Border, D. J., Buck, K. W., Chain, E. B., Kempson-Jones, G. F., Lhoas, P. \& Ratti, G. (1972). Viruses of Penicillium and Aspergillus species. Biochemical Journal 127, 4 P-6P.

Bozarth, R. F., Wood, H. A. \& Mandelbrot, A. (I97I). The Penicillium stoloniferum virus complex: two similar double-stranded RNA virus-like particles in a single cell. Virology 45, 516-523.

Buck, K. W. \& Kempson-Jones, G. F. (1973). Biophysical properties of Penicillium stoloniferum virus S. Journal of General Virology 18, 223-235.

ColE, G. T. \& KENDRICK, W. B. (I969). Conidium autogeny in hyphomycetes. The phialides of Phialophora, Penicillium and Ceratocystis. Canadian Journal of Botany 47, 779-789.

Detroy, R. W., LillehoJ, E. B. \& Hesseltine, C. W. (1974). Replication of virus-like particles in Penicillium stoloniferum mycelia. Canadian Journal of Microbiology 20, I 1 3-117.

ElLis, L. F. \& KLeINSCHMidT, W. J. (1967). Virus-like particles of a fraction of statolon, a mould product. Nature, London 215, 649-650.

FLetCHer, J. (I97I). Conidium autogeny in Penicillium. Journal of General Microbiology 67, 207-214.

Hollings, M. \& Stone, O. M. (197I). Viruses that infect fungi. Annual Review of Phytopathology 9, 93-I I 8.

Hooper, G. R., Wood, H. A., Myers, R. \& Bozarth, R. F. (1972). Virus-like particles in Penicillium brevicompactum and $P$. stoloniferum hyphae and spores. Phytopathology 62, 823-825.

LhoAs, P. (1971). Transmission of double stranded RNA viruses to a strain of Penicillium stoloniferum through heterokaryosis. Nature, London 230, 248-249.

LoENING, U. E. (1967). The fractionation of high-molecular-weight ribonucleic acid by polyacrylamide-gel electrophoresis. Biochemical Journal 102, 25I-257.

Sansing, G. A., Detroy, R. W., Freer, S. N. \& Hesseltine, C. W. (1973). Virus particles from conidia of Penicillium species. Applied Microbiology 26, 914-9I 8.

Still, P. E., Detroy, R. W. \& Hesseltine, C. W. (I975). Penicillium stoloniferum virus: altered replication in ultraviolet-derived mutants. Journal of General Virology 27, 275-28I.

Volterra, L., Cassone, A., Tonolo, A. \& Bruzzone, M. L. (I975). Presence of two virus-like particles in Penicillium citrinum. Applied Microbiology 30, I49-15I.

ZaChariah, K. \& FITZ-J AMES, P. C. (I967). The structure of phialides in Penicillium claviforme. Canadian Journal of Microbiology 13, 249-256 (pl. I-VIII). 